\title{
Direct detection of OXA-48 carbapenemase gene in lysate samples through changes in mechanical properties of DNA monolayers upon hybridization.
}

\author{
Carmen M. Domínguez, ${ }^{* \dagger 1}$ Daniel Ramos, ${ }^{1}$ Jesús Mingorance, ${ }^{2}$ José L. G. Fierro, ${ }^{3}$ Javier Tamayo, ${ }^{1}$ \\ Montserrat Calleja. ${ }^{1}$ \\ 1 IMN-Instituto de Micro y Nanotecnología (CNM-CSIC), Isaac Newton 8, PTM, E-28760 Tres Cantos, Madrid, Spain.2 \\ Servicio de Microbiología, Hospital Universitario La Paz, IdiPAZ, Paseo de la Castellana, 261, E-28046 Madrid, Spain \\ 3 ICP-Instituto de Catálisis y Petroleoquímica (CSIC), Marie Curie, 2. Cantoblanco, E-28049, Madrid, Spain.
}

\begin{abstract}
Carbapenem-resistant Enterobacteriaceae have become recently an important cause of morbidity and mortality due to healthcare-associated infections. Most commonly used diagnostic methods present time-to-result incompatible with fast and accurate directed therapy. We report here the direct identification of the blaOXA48 gene, which codes for the carbapenemase OXA-48, in lysate samples from Klebsiella pneumoniae. The method is PCR-free and label-free. It is based on the measurement of changes in the stiffness of DNA self-assembled monolayers anchored to microcantilevers that occur as a consequence of the hybridization. The stiffness of the DNA layer is measured through changes of the sensor resonance frequency upon hybridization and at varying relative humidity.
\end{abstract}

The global spread of drug resistant pathogens has been recently identified as a major public health concern. ${ }^{1}$ By 2050 more than 10 million deaths all over the world are expected due to these microorganisms. ${ }^{2}$ Among them, carbapenemresistant Enterobacteriaceae have become resistant to most available antibiotics and have been globally reported. ${ }^{3-5}$ The Enterobacteriaceae are among the most abundant commensal microorganisms in the human body and the main cause of serious nosocomial infections in humans. ${ }^{6}$ Carbapenem is commonly used as a last resort antibiotic when infections are produced by $\beta$-lactamase-producing organisms and other antibiotics are not effective. Though there are different mechanisms responsible for the resistance to carbapenems, such as porin loss or overexpression of drug excretion systems, carbapenemase production is the most important mechanism. ${ }^{7}$ Carbapenemase producers are resistant to all or most $\beta$-lactams and usually associate resistance to most of the non-betalactams antibiotics, leaving very few therapeutic options to treat hospitalized patients, to which they mainly affect, and leading to high mortality rates. ${ }^{8}$ Tackling the fast detection of these resistant strains is of paramount importance to prevent their evolution, dissemination and transmission.

Most commonly used diagnostic methods rely on the detection of phenotypic resistance on bacterial cultures. ${ }^{9}$ There are different classes of carbapenemases. OXA-48 is a prevalent carbapenemase that has caused outbreaks of antibiotic resistant bacteria worldwide. ${ }^{10}$ This carbapenemase is encoded by the blaxA48 gene, which is carried and transferred by a $63 \mathrm{~kb}$ plasmid. OXA-48 producers are likely the most difficult carbapenemase producers to be identified by phenotypic techniques due to their particular combination of susceptibilities and resistances and the slight increases in carbapenem MICs (minimum inhibitory concentrations). ${ }^{11}$ Phenotypic methods are often time-consuming (results may delay up to $18 \mathrm{~h}$ ), thus the time needed to yield results is incompatible with a fast treatment decision. Moreover, these tests cannot discriminate types within each class of carbapenemases and they are also unable to confirm in a single test the occurrence of OXA-48 carbapenemase. ${ }^{12-15}$ Thus, a molecular approach to detect OXA-48 producers is required. Molecular diagnostic methods, such as conventional PCR and RT-PCR, are more reliable for carbapenemase identification due to their high sensitivity and specificity. ${ }^{16-19}$ Still, the susceptibility to contamination and the polymerase reaction inhibition by molecules present in clinical samples (e.g. haemoglobin, heparin, IgG, lactoferrin, among others) affect their efficiency and the limits of detection achieved. ${ }^{20-21}$ Moreover, the time needed to obtain the results with conventional PCR ranges from 2 to $6 \mathrm{~h} .13,{ }^{22} \mathrm{RT}-\mathrm{PCR}$ is faster but also more costly. ${ }^{23}$ Addressing effective detection of OXA-48 necessarily implies the development of a specific, fast, portable and cheap biosensing device. PCR-free and label-free novel methodologies for the detection of carbapenemase producers are under development. For instance, methods based on mass spectrometry (MALDITOF), ${ }^{24-25}$ electrochemistry ${ }^{15}$ or immunochromatography. ${ }^{26}$ However, they have not been established yet as alternative techniques to the most used methods. ${ }^{13}$

Microcantilevers have proven to be highly sensitive label-free biosensors for microbial DNA detection ${ }^{27-29}$ and have the potential for developing instruments suitable for both laboratory and point-of-care testing. Here we report the labelfree and PCR-free detection of bla OXA48 $_{4}$ gene using total extracted DNA from carbapenem resistant Klebsiella 
pneumoniae (KP) cultures. We apply concepts recently demonstrated with synthetic oligonucleotides, ${ }^{30}$ demonstrating that the differences in the mechanical properties of ssDNA probe layers and their hybridized counterparts, measured experimentally as frequency shifts, can be harnessed to discriminate the presence of specific long DNA fragments (200-300 bp) belonging to bla $a_{\text {OxA48 }}$ gene in complex samples.

\section{EXPERIMENTAL SECTION}

Surface functionalization. Arrays of eight silicon microcantilevers with $20 \mathrm{~nm}$ gold coating were purchased from Micromotive (Germany). The cantilevers are $500 \mu \mathrm{m}$ long, $90 \mu \mathrm{m}$ wide and $1 \mu \mathrm{m}$ thick. Since the cleanliness of the gold surface affects the mechanical response of the cantilevers, prior to its use, cantilever arrays were deeply cleaned by two consecutive procedures that remove organic contaminants. They were immersed in acetone and isopropyl alcohol; dried under a stream of dry nitrogen and irradiated in a UV-Ozone cleaner for 1 hour.

$K$. pneumoniae duplicates each 1 hour approximately in solid rich medium during exponential phase ${ }^{31}$ and clinical samples are often obtained from culture. Since the growth rate is not a limiting factor for this pathogen, we have focused our efforts in achieving good sensitivity and specificity rather than an ultra-low limit of detection. For this purpose, we have designed three probe sequences, OXA48_p1, OXA48_p2 and OXA48_p3 (see Table S1 in Supporting Information), that were immobilized onto the gold-coated microcantilevers. The three probe sequences were diluted in the degassed immobilization buffer and mixed in 1:1:1 proportions to a final DNA concentration of $5 \mu \mathrm{M}$. The cantilever arrays were incubated in the probe DNA solution for $135 \mathrm{~min}$. The incubation was carried out at $25^{\circ} \mathrm{C}$ under agitation. Afterwards, the arrays were cleaned with wash buffers WB1 and WB2 (see Supporting Information) for 5 and 20 minutes, respectively, to wash out the physisorbed DNA away from the microcantilever surface and finally rinsed with plenty of Milli$\mathrm{Q}$ water. Cleansing steps were carried out at $25^{\circ} \mathrm{C}$ as well. In order to determine the packing density of DNA probes at the cantilever surface we have performed a quantitative characterization of the DNA film by X-ray photoelectron spectroscopy (XPS). See S4 in Supporting Information for more details.

Preparation of target DNA and hybridization conditions. Several clinical isolates were obtained from the culture collection of the Microbiology Department of Hospital La Paz (Madrid, Spain) from OXA-48 positive and OXA-48 negative KP cultures. OXA-48+ isolates are carbapenemase producers, hence resistant to carbapenems, and OXA-48- isolates are susceptible to carbapenems, since they do not produce carbapenemase enzymes. Several KP colonies were taken from blood agar plates, resuspended in phosphate buffered saline (PBS) and boiled for 15 minutes. The experiments were performed with different samples: one sample type contained purified DNA and the other one, boiled bacterial lysates with the DNA mixed with bacterial debris. The UltraClean ${ }^{\circledR}$ Microbial DNA Isolation Kit (MoBio) was used for DNA purification.

The DNA concentrations were measured with a spectrophotometer, NanoDrop 2000 (Thermo Fisher), by means of its absorbance at $260 \mathrm{~nm}$. The DNA was partially digested with the restriction enzyme FastDigest Hin6I (Thermo Fisher) following the recommendations given by the manufacturer. The FastDigest Hin6I restriction enzyme recognizes $\mathrm{G}^{\wedge} \mathrm{CGC}$ sites and splits the blaOXA48 gene in three fragments, being each of them complementary to one of the probe sequences. Prior to hybridization, the DNA was denatured at $95{ }^{\circ} \mathrm{C}$ for $5 \mathrm{~min}$ and placed on ice for $2 \mathrm{~min}$. Then, it was diluted directly to a final DNA concentration of $200 \mathrm{ng} / \mathrm{mL}$ in TE-NaCl $0.5 \mathrm{mM}$ buffer and mixed vigorously to homogenize the solution. Hybridization was performed at $30{ }^{\circ} \mathrm{C}$ for $45 \mathrm{~min}$ under agitation. After hybridization, the cantilever arrays were cleaned with low and high stringency hybridization wash buffers (WB1 and WB2) for 5 and 15 minutes, respectively, and extensively rinsed with Milli-Q water. The higher stringency wash was carried out at the hybridization temperature $\left(30^{\circ} \mathrm{C}\right)$, while the lower stringency wash was performed at $25^{\circ} \mathrm{C}$.

Measurement setup and experiments performed. The experiments for the detection of the bla OxA48 $_{4}$ gene have been addressed through the measurement of both the surface stress and the fundamental resonance frequency shift of microcantilevers functionalized with the probe DNA upon hydration before and after incubation with samples coming from resistant $\left(\mathrm{OXA}-48^{+}\right)$and susceptible $\left(\mathrm{OXA}-48^{-}\right) \mathrm{K}$. pneumoniae isolates. Figure 1 shows a depiction of the experiments carried out. The measurements were performed simultaneously in homebuilt equipment with capability for read-out of single chips of eight cantilevers. The read-out is based on the optical beam deflection method. A laser is focused onto the free end of the cantilever beam and its reflection is collected by a position sensitive detector (PSD). In order to prevent artifacts in the resonance shifts due to the non-uniform response of the PSD over its total surface, we have introduced in the optical path two mirrors actuated by an automatized motor in a feedback closed loop configuration, so the laser spot is centered on the PSD at any time. ${ }^{30}$ The input signal of the mirror angle is translated into the static deflection of the cantilever, whereas the resonance frequency is obtained by the Fast Fourier Transform (FFT) of the signal from the PSD and it is free from undesirable artifacts (see reference 30 for further details). The system was equipped with an environmental chamber with the capability to keep the temperature at $25.00 \pm 0.02{ }^{\circ} \mathrm{C}$ and to change relative humidity at a rate of $10.00 \pm 0.08 \% \mathrm{~min}^{-1}$.

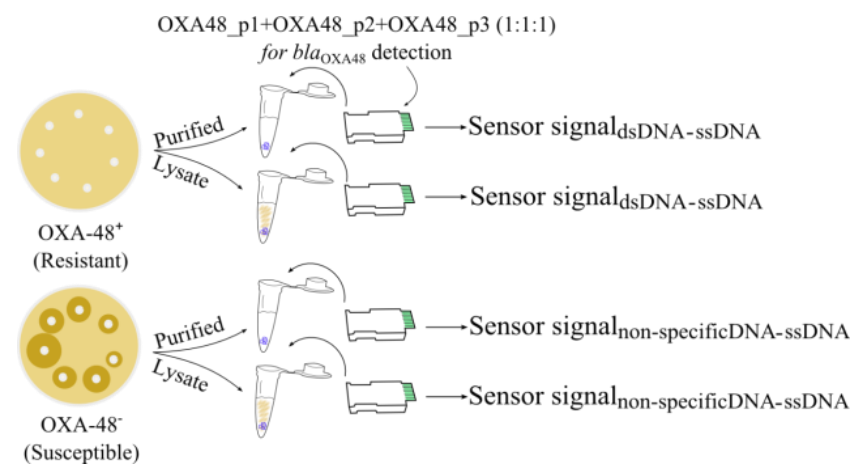

Figure 1. Depiction of the experiments performed for the detection of the plasmid harboring the bla $a_{\mathrm{OxA} 48}$ gene. 
To account for the change of the thickness of the DNA layers, that in turn affects the mechanical properties of the cantilevers, we have characterized gold coated surfaces selectively immobilized with ssDNA and those after hybridization by means of Atomic Force Microscopy (AFM). See S5 in Supporting Information for details.

\section{RESULTS AND DISCUSSION}

Static mode. Microcantilevers sensitized with probe ssDNA measured in the static mode have proven successful ultrasensitive detection of purified genomic DNA. ${ }^{27}, 32$ Specifically, the measurement of the surface stress upon hydration before and after hybridization has been used by the authors to detect the pathogen Mycobacterium tuberculosis and to discriminate the presence of a single mismatch with high sensibility and specificity. ${ }^{27}$ Given the good results achieved working with the static mode, as a first approach we have attempted to detect the plasmid measuring the difference in the surface stress generated with hydration after hybridization. For details, see references ${ }^{27,33}$.

Figure 2 shows the representative surface stress curves obtained with a relative humidity cycle for the microcantilevers sensitized with the mixture of ssDNA probes (black line in a) and b)) and after incubation with purified DNA from OXA-48 ${ }^{+} \mathrm{KP}$ isolates (green line in a)), which contain the plasmid harboring the bla oxA48 gene; and OXA-48 $\mathrm{KP}$ isolates (red line in b)). In order to reduce the preparation time, the immobilization time of the ssDNA probes has been reduced to $135 \mathrm{~min}$, as mentioned in the experimental section. Note that immobilization for ex situ static nanomechanical measurements has been commonly carried out overnight. ${ }^{27,}$,33${ }^{34}$ Given this, the surface densities achieved, measured by XPS as described above, range from 1.4 to $7.2 \mathrm{x} 10^{12}$ molecules $/ \mathrm{cm}^{2}$. In-plane forces between the tethered ssDNA strands, generated by changes in the conformational entropy due to their hydration, result in an effective change of the cantilever surface stress that gives rise to the cantilever bending. The interaction forces, and thus the surface stress, critically depend on the conformation of the DNA layers. ${ }^{33,35}$ In order to reduce the analysis time, and thus increase the interest for clinical applications of the device, the hybridization time has been reduced to $45 \mathrm{~min}$. At the probe densities mentioned above, this hybridization time allows the molecules involved to complete the formation of the double helix, resulting in an increase of the persistence length of the DNA molecules. These layers formed by stiffer rods give rise to a decrease of the surface stress, with respect to the ssDNA layers response, as can be seen in Figure 2 a).

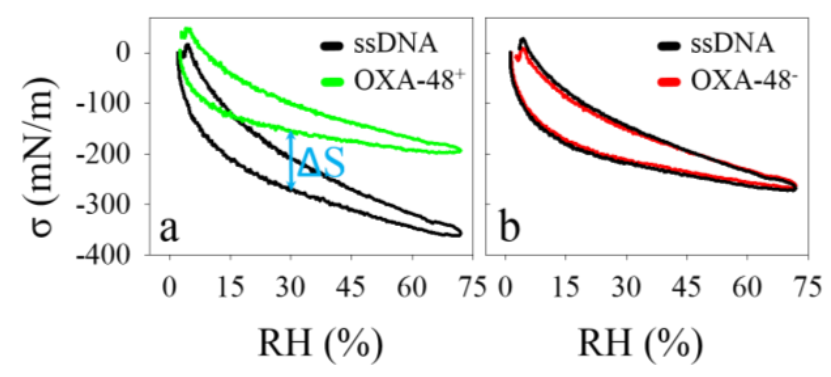

Figure 2. Representative curves of microcantilever surface stress upon hydration before and after incubation with purified DNA from a) OXA- $48^{+} \mathrm{KP}$ isolates (green line), which contain the plasmid harboring the bla oxA48 gene; and b) OXA$48^{-} \mathrm{KP}$ isolates (red line), which do not contain the plasmid

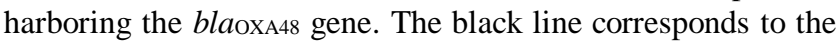
response of the microcantilevers sensitized with the mixture of the ssDNA probes.

The sensor signal in static mode, $\Delta \mathrm{S}$, has been defined as described previously ${ }^{33}$ (see $\Delta \mathrm{S}$ in Figure 2). Figure 3 shows the sensor signal $\Delta \mathrm{S}$ obtained for the microcantilevers incubated with extracted and purified DNA samples from OXA $-48^{+}$(green circles) and OXA-48- (red circles) KP isolates; and for the microcantilevers incubated with bacterial lysate samples from OXA- $48^{+}$(green hexagons) and OXA-48 (red hexagons) KP isolates. By defining the detection threshold value for the sensor signal $\Delta \mathrm{S}$ as the mean value of the signals obtained for the OXA-48- samples plus twice the standard deviation, in the purified DNA assay the rate of true positives and true negatives in this small pool of tests (50 cantilevers, belonging to 8 arrays) is 0.90 and 0.93 , respectively; while in the bacterial lysate assay is 0 and 1 , respectively. Hence, though the method based on the measurement of the surface stress allows to discriminate the presence of the bla $\mathrm{OXA}-48$ gene in a sample containing purified and digested DNA, in the present experimental conditions it is

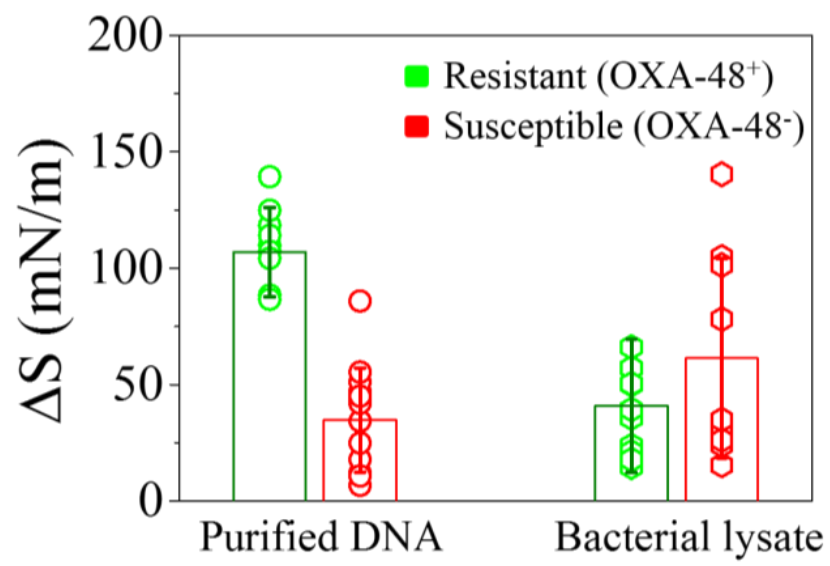

not able to detect the gene in the more challenging cellular lysate sample.

Figure 3. Nanomechanical static detection of the plasmid harboring the bla $\mathrm{OXA}-48$ gene from KP. It is shown the average value and standard deviation of the sensor signal $\Delta \mathrm{S}$ obtained for the microcantilevers incubated with extracted and purified plasmid samples from OXA- $48^{+}$(green circles) and OXA-48 (red circles) KP isolates; and for the microcantilevers incubated with bacterial lysate samples from OXA- $48^{+}$(green hexagons) and OXA-48- (red hexagons) KP isolates.

Dynamic mode. Both dynamic and static measurements were done simultaneously. Figure 4 a) and b) shows the representative resonance frequency curves obtained under a relative humidity cycle for the microcantilevers sensitized with the ssDNA probes (black line in a) and b)) and after incubation with purified DNA from OXA $-48^{+} \mathrm{KP}$ isolates (green line in a)), which contain the plasmid harboring the 


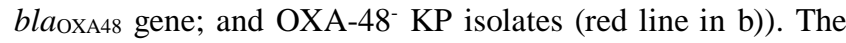
shadowed area represents the standard deviation obtained for the 8 microcantilevers measured within one array. The curves are referenced to the initial dry state.

We have shown recently that intermolecular forces in DNA layers upon hydration give rise to a collective elastic behaviour, which in turn affects the resonance frequency of the microcantilevers. ${ }^{30}$ The resonance frequency of a cantilever shifts as a consequence of changes in the effective mass and stiffness of the resonator. The effective stiffness is affected by changes in both the thickness and the elastic properties of the biological layer adsorbed onto the cantilever. Young's modulus can be understood as the result of the sum of the intermolecular forces acting in the layer. As intermolecular forces depend directly on intermolecular distances, the stiffness effect is also surface-density dependent. Moreover, these forces are largely affected by the hydration state, as water molecules intercalate between DNA strands and mediate DNA-DNA interactions. As mentioned above, the probe densities used here are between 1.4 and $7.2 \mathrm{x}$ $10^{12}$ molecules $/ \mathrm{cm}^{2}$. At these low packing densities, due to the large intermolecular distances, the contribution of DNA-DNA intermolecular forces, and thus, both the Young's modulus and the thickness of the layer, do no dominate the resulting resonance frequency of the system. Therefore, hydration of the ssDNA layer shifts the resonance frequency towards lower values, compared to the bare gold response, due to the added mass of water molecules that increase the effective mass of the resonator (black lines in Figure 4 a) and b). As we can see in Figure 4 , the frequency decreases $\sim 180 \mathrm{mHz}$ for the cantilevers shown in a) and $\sim 100 \mathrm{mHz}$ for those shown in b). We attribute the variability in the frequency shift upon hydration among different microcantilever (shadowed area) to differences in the packing density. As hybridization increases the persistence length of the DNA and has a shielding effect over the intermolecular interactions between ssDNA strands, both the thickness and the Young's modulus change with the transition from ssDNA to dsDNA layer. This difference in the mechanical properties, as well as differents in the adsorption of water molecules from both ssDNA and dsDNA layer, results in a frequency shift that allows detecting target DNA molecules by their hybridization with the probe DNA layer. ${ }^{30}$ We have previously demonstrated the detection of target oligonucleotides by measuring the frequency shift upon hydration of microcantilevers sensitized with probe ssDNA before and after incubation with the complementary sequences. ${ }^{30}$ Here, we have applied the methodology based on the measurement of the frequency shift upon hydration to the direct detection of the bla $a_{\text {OXA-48 }}$ gene in bacterial lysate samples, eliminating the need for DNA purification.
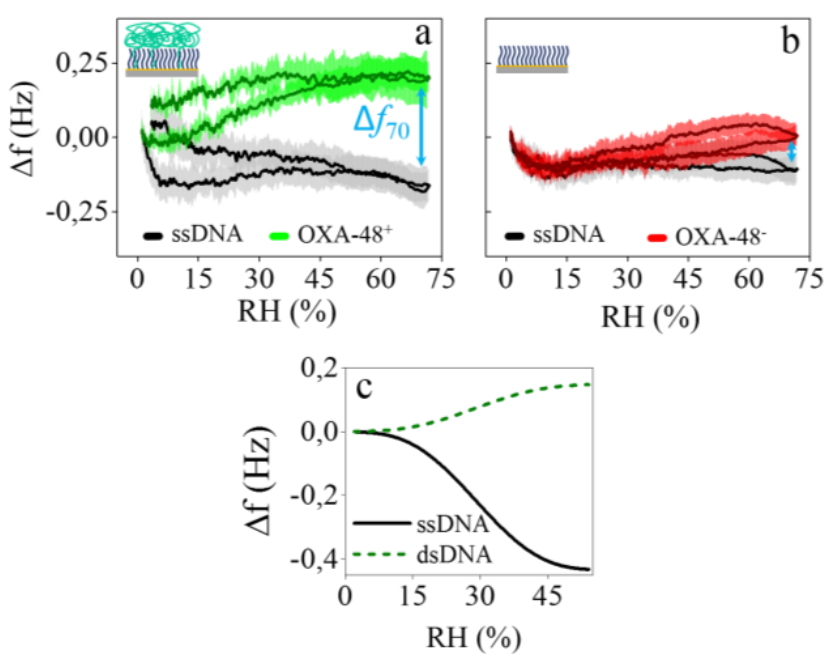

Figure 4. Representative curves of microcantilever frequency shift upon hydration before and after incubation with purified plasmids from a) OXA- $48^{+} \mathrm{KP}$ isolates (green line), which contain the plasmid harboring the blaOXA48 gene; and b) OXA- $48^{-} \mathrm{KP}$ isolates (red line). The black line corresponds to the response of the microcantilevers sensitized with the ssDNA probes. Cantilevers incubated with OXA- $48^{+}$samples show a frequency shift of $400 \mathrm{mHz}$ at the maximum $\mathrm{RH}$. Cantilevers incubated with OXA- $48^{-}$samples show a frequency shift of $100 \mathrm{mHz}$, within the measurement error and mainly due to non-specific adsorption. The inset depictions represent the status of the (a) hybridized DNA layer and the (b) non-hybridized DNA layer after incubation with the plasmidic DNA. The curves are referenced to the dry state. c) Finite element simulations of the relative frequency shift as a function of the relative humidity. The black solid line corresponds to the probe ssDNA, whereas the green dashed line represents the frequency shift after incubation with the target molecule.

The DNA molecules targeted here are between $200-300$ bases in length, while the probe ssDNA molecules are 30 bases long. After hybridization, since it occurs only in 30 bases of the target DNA, we hypothesize most of each target molecule lies over the DNA probe layer at the dry state (see schematics inset in Figure 4 a). With hydration, the polyelectrolyte swells and the persistence length decreases, giving rise to an increase of the intermolecular forces and thus of the thickness. Hence, due to this thickness increase upon hydration, the stiffness effect dominates over the mass effect and the resonance frequency goes to higher values with $\mathrm{RH}$. As Figure 4 a shows, the resonance frequency of the hybridized cantilevers increases $\sim 200 \mathrm{mHz}$ at the maximum relative humidity $(70 \%)$, giving a difference between the ssDNA and the hybridized layer of about $380 \mathrm{mHz}$. In Figure $4 \mathrm{~b}$ ) we see a difference in the resonance frequency of about $100 \mathrm{mHz}$, that is within the measurement error and can be attributed to unspecific hybridization or adsorption. 
To prove the consistency of our hypothesis we have measured the thicknesses of the DNA layers before and after hybridization and at two different relative humidities (2\% and $54 \%$ ) by AFM (see S5 in Supporting Information); and we have performed theoretical simulations to account for the shift in frequency considering the thickness variations measured. For the theoretical simulations we have used a combination of finite element simulations (FEM) and molecular dynamics (MD) as described in reference. ${ }^{30}$ By using the expressions described in previous work in literature, we have all the necessary elements to simulate the response of a nanomechanical resonator with an attached effective polymerlike layer varying its thickness, Young's modulus and mass density as function of the surrounding relative humidity. Figure $4 \mathrm{c}$ ) shows the FEM simulations of a cantilever with the same dimensions than the experimental device $(500 \mu \mathrm{m}$ long, $90 \mu \mathrm{m}$ wide and $1 \mu \mathrm{m}$ thick). In black solid line, we can see the effect on the resonance frequency of increasing relative humidity in ssDNA layer on top of the cantilever. In order to mimic the experimental conditions, the molecular surface probe density used in this case is of $4 \times 10^{12}$ molecules $/ \mathrm{cm}^{2}$, with a thickness of $2.5 \mathrm{~nm}$ with a variation two times lower with relative humidity than that obtained for the hybridized layer, as indicated by our AFM measurements (supplementary material). The Young's modulus of the ssDNA layer asymptotically grows until a saturation value of $12 \mathrm{GPa}$ with increasing humidity. The negative frequency shift shown in the simulation is mainly due to the added mass of the water molecules during the hydration cycle. We have also calculated the relative frequency shift corresponding to the simulated absorption of the target layer, which is also shown in Figure 4 c) as green dashed line. In this case, we have considered the variation of the thickness along the hydration cycle ranges from $2.8 \mathrm{~nm}$ at low $\mathrm{RH}$ to $3.3 \mathrm{~nm}$ at the maximum $\mathrm{RH}$, as obtained through the complementary AFM experimental measurements. From previous work, we can expect that the hybridized part of the probe strands of $30 \mathrm{bp}$ leads to an the effective Young's modulus of the dsDNA layer is one order of magnitude lower than the single stranded layer due to the shielding effect, as shown in reference ${ }^{30}$, however since the length of the target molecule is 10 times larger than the ssDNA probe, most of the strand remains unhybridized over the ssDNA probe layer, behaving like a single stranded molecule that swells with hydration (see references ${ }^{33}$ and ${ }^{36}$ ). Thus, similarly to the ssDNA layer the Young's modulus of the hybridized layer asymptotically grows until a saturation value of $11 \mathrm{GPa}$ with hydration (see supplementary materials,). This single-stranded region of the target is responsible for the observed thickness variations with RH by AFM. On the contrary, the dsDNA monolayers of oligonucleotides of comparable length to the target molecules show negligible variation with relative humidity, due to the increased stiffness of the dsDNA molecule and the shielding effect of the double helix conformation for the inter-strand forces. ${ }^{30}$

As this different mechanical behaviour of the resonator at the fully hydrated state as a function of the hybridization has allowed to discriminate the presence of the blaxA-48 gene in samples containing purified DNA, we have arbitrarily defined our sensor signal, $\Delta f_{70}$, as the difference of the resonance frequency value at $70 \% \mathrm{RH}$ between the ssDNA hydration curve and the curve obtained after the incubation with the KP sample, $\quad \Delta f_{70}=\Delta \boldsymbol{f}_{\boldsymbol{d S D N A , 7 0} \% \boldsymbol{R H}}-\Delta \boldsymbol{f}_{\boldsymbol{s S D N A , 7 0} \% \boldsymbol{R H}}$. Once defined our sensor signal, we have proceeded to the analysis of the same samples analyzed by the surface stress method.

We first performed the detection experiments with samples containing extracted DNA. Once achieved this, we proceeded with the bacterial lysates, in which the DNA is embedded in excess of bacterial debris. In figure 5 we show the average value and standard deviation of the sensor response signal $\Delta f_{70}$ obtained for the cantilevers incubated with purified DNA from OXA- $48^{+}$(green circles) and OXA-48- (red circles) KP isolates; and for the microcantilevers incubated with bacterial lysate samples from OXA-48+ (green hexagons) and OXA-48 (red hexagons) KP isolates. By defining the detection threshold value for the sensor signal $\Delta f_{70}$ as the mean value of the signals obtained for the OXA- $48^{-}$samples plus twice the standard deviation, in the purified plasmid assay the rate of true positives and true negatives is 1 and 1, respectively; while in the bacterial lysate assay is 0.73 and 1 , respectively in a small pool of measurements of 50 cantilevers ( 8 arrays).

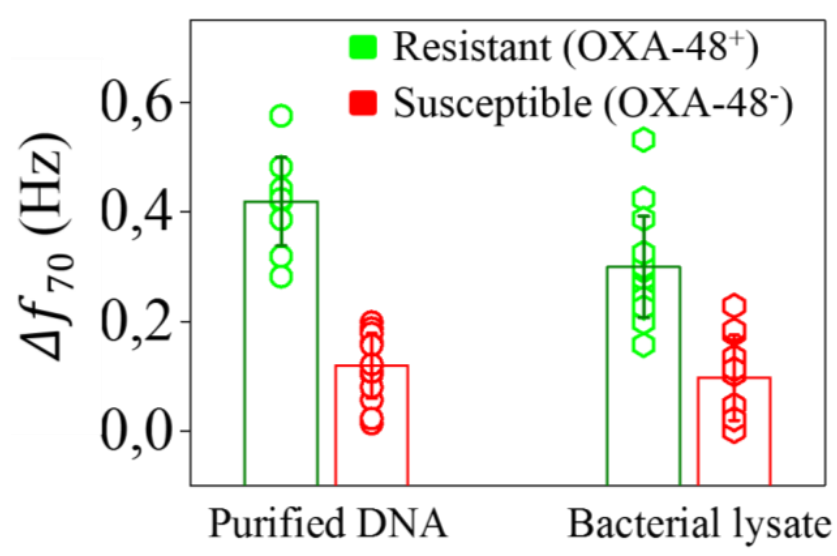

Figure 5. Nanomechanical dynamic detection of the plasmid harboring the bla $a_{\text {OXA-48 }}$ gene from KP. It is shown the average value and standard deviation of the sensor signal $\Delta f_{70}$ obtained for the microcantilevers incubated with extracted and purified plasmid samples from OXA- $48^{+}$(green circles) and OXA-48 (red circles) KP isolates; and for the microcantilevers incubated with bacterial lysate samples from the same OXA$48^{+}$(green hexagons) and OXA-48- (red hexagons) KP isolates.

The ex-situ measurement of both the surface stress and the stiffness variation upon hybridization of hydrated DNA layers tethered to microcantilevers allows the successful discrimination of the bla $a_{\mathrm{OXA}} 48$ gene in the presence of nonrelated DNA (purified DNA samples) coming from $K$. pneumoniae cultures. The blaoxA48 gene has been detected in a total analysis time of $1 \mathrm{~h} 30 \mathrm{~min}$. This is very advantageous since commonly used diagnostic phenotypic methods for the detection of carbapenemase producers require up to $16-18 \mathrm{~h}$ to get the results ${ }^{18,} 37$ and molecular methods such as conventional PCR may delay more than 4 h. ${ }^{13,22}$ Real time PCR allows obtaining immediate results but multiplexed detection is challenging and it is a more costly technique. ${ }^{23}$

The methodology presented here based on the dynamic ex-situ measurements of the cantilevers allows to discriminate the 


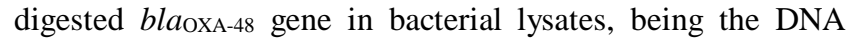
embedded in excess of bacterial debris, with a rate of true positives and true negatives of 0.73 and 1 , respectively. The direct detection of the DNA in this kind of sample is challenging and, to the extent of our knowledge, this is the first time a nanomechanical sensor successfully detects DNA in lysate samples with no prior purification.

We have also observed the method based on the static characterization of the cantilevers does not have the capability to detect the plasmidic DNA in a mixture of bacterial debris with the established protocol. The generation of a differential surface stress is due to a collective phenomenon that requires a minimum receptor coverage. The connection between regions where molecular recognition happens is critically needed for the proper transmission of the surface stress and the generation of the bending. ${ }^{38}$ Even if the proportion of hybridized targets is low, the molecular crowding effect due to a high packing density in the probe layer allows to transduce efficiently the surface stress, as demonstrated before. ${ }^{27}$ However, and given the inherent difficulty of attaining homegeneous and highly packed ssDNa monolayers, in this study we have attempted to reproduce the more attainable technological situation, being the packing probe density between 1.4 and $7.2 \times 10^{12}$ molecules $/ \mathrm{cm}^{2}$. Thus, the lower molecular crowding state obtained is likely responsible for the inefficient transmission of the surface stress generated along the hybridized layers. We show here that at low probe densities static measurements fail to detect the target DNA in cell lysate samples. However, due to the high sensitivity to small changes of the thickness, dynamic measurements allow to discriminate the plasmid in a complex sample. The hybridization efficiency suffices for the DNA detection in lysate samples through the measurement of the resonance frequency shift. In this case, the stiffness change, arising due to the thickness variations with $\mathrm{RH}$ in the dsDNA layers allow the hybridization detection. The obtained rate of true positives of 0.73 , indicates that the detection protocol must be improved in order to gain sensitivity in the assay. Lysate clearing by centrifugation or protein precipitation, longer digestion times and/or the use of volume excluders might be considered to increase the local concentration of the complementary DNA fragments without adding complexity to the assay or increasing the time analysis. A reduction of the hybridization stringency by lowering the hybridization temperature and/or increasing the $\mathrm{Na}^{+}$ concentration should also be considered.

\section{CONCLUSIONS}

This is the first time a nanomechanical sensor is used to detect cellular DNA in a lysate sample without prior purification. We have demonstrated the detection of carbapenemase OXA-48 producing $K$. pneumoniae at the proof of concept level by means of the detection of the codifying gene (bla $a_{\text {oxA48) }}$ through the measurement of the resonance frequency shift of microcantilevers upon hydration. Since there is no need for achieving high packing densities of the probe layer, the methodology is easily implementable with commercial gold coatings or even recycled surfaces, with which obtaining highly packed DNA layers is challenging. Though the technique presents high sensitivity and specificity to discriminate the bla $a_{\text {OXA48 }}$ gene in the presence of non-related DNA in excess, the sensitivity achieved with bacterial lysates needs to be further improved for the clinical application of the technology. This technique has the potential for multiplexed detection and miniaturization. The reduction of the sample preparation steps is of utmost importance to move forward on the development of fast and easy-to-manage laboratory devices for antimicrobial resistance detection. Thus, we believe the direct detection of DNA in lysate samples with no prior purification, amplification or labelling demonstrated here paves the way to the development of a fast and easy-tomanage device based on nanomechanical biosensing.

\section{ASSOCIATED CONTENT}

\section{Supporting Information}

The Supporting Information is available free of charge on the ACS Publications website.

DNA probe sequences (Table S1), reagents (S2), buffers and solutions (S3), characterization of the DNA immobilization density by X-ray photoelectron spectroscopy (XPS) measurements (S4), characterization of ssDNA and dsDNA monolayers by Atomic Force Microscopy (S5), AFM characterization of the thickness of the DNA layers (Figure S5), (PDF).

\section{AUTHOR INFORMATION}

\section{Corresponding Author}

* carmen.dominguez@kit.edu

\section{Present Addresses}

† IBG 1-Institut für Biologische Grenzflächen 1, Karlsruher Institut für Technologie (KIT), Hermann-von-Helmholtz-Platz 1, D-76344 Eggenstein-Leopoldshafen, Karlsruhe, Germany

\section{Author Contributions}

The manuscript was written through contributions of all authors. All authors have given approval to the final version of the manuscript.

\section{ACKNOWLEDGMENT}

This work was supported by the Spanish Science Ministry (MINECO) through project MAT2015-66904-R, and by European Research Council through NANOFORCELLS project (ERC-StG2011-278860) and European Union's Horizon 2020 research and innovation program under grant agreement No 731868 VIRUSCAN. D.R. acknowledges the Ramon y Cajal fellowship supported by Spanish Ministry MINECO. Authors also acknowledge the service from the X-SEM Laboratory at IMM, and funding from MINECO under project CSIC13-4E-1794 with support from EU (FEDER, FSE).

\section{REFERENCES}

1. Stadler, M.; Dersch, P., How to Overcome the Antibiotic Crisis: Facts, Challenges, Technologies and Future Perspectives. Springer: 2016; Vol. 398.

2. Resistance, A., Tackling a Crisis for the Health and Wealth of Nations. The Review on Antimicrobial Resistance, Chaired by Jim O'Neill. December 2014. 2015.

3. Nordmann, P.; Dortet, L.; Poirel, L., Carbapenem resistance in Enterobacteriaceae: here is the storm! Trends in molecular medicine 2012, 18 (5), 263-272.

4. Hawkey, P., The growing burden of antimicrobial resistance. Journal of antimicrobial chemotherapy 2008, 62 (suppl 1), i1-i9. 
5. Pitout, J. D.; Laupland, K. B., Extended-spectrum $\beta$ lactamase-producing Enterobacteriaceae: an emerging publichealth concern. The Lancet infectious diseases 2008, 8 (3), 159-166.

6. Bennett, J. E.; Dolin, R.; Blaser, M. J., Principles and practice of infectious diseases. Elsevier Health Sciences: 2014.

7. Pitout, J. D.; Nordmann, P.; Poirel, L., Carbapenemase-producing Klebsiella pneumoniae, a key pathogen set for global nosocomial dominance. Antimicrobial agents and chemotherapy 2015, 59 (10), 5873-5884.

8. Grundmann, H.; Livermore, D.; Giske, C.; Canton, R.; Rossolini, G.; Campos, J.; Vatopoulos, A.; Gniadkowski, M.; Toth, A.; Pfeifer, Y., Carbapenem-non-susceptible Enterobacteriaceae in Europe: conclusions from a meeting of national experts. Eurosurveillance 2010.

9. Pulido, M. R.; García-Quintanilla, M.; Martín-Peña, R.; Cisneros, J. M.; McConnell, M. J., Progress on the development of rapid methods for antimicrobial susceptibility testing. Journal of Antimicrobial Chemotherapy 2013, dkt253. 10. Poirel, L.; Potron, A.; Nordmann, P., OXA-48-like carbapenemases: the phantom menace. Journal of Antimicrobial Chemotherapy 2012, 67 (7), 1597-1606.

11. Nordmann, P.; Poirel, L.; Dortet, L., Rapid detection of carbapenemase-producing Enterobacteriaceae. Emerg Infect Dis 2012, 18 (9), 1503-1507.

12. Doi, Y.; Paterson, D. L. In Carbapenemaseproducing Enterobacteriaceae, Seminars in respiratory and critical care medicine, Thieme Medical Publishers: 2015; pp 074-084.

13. Matsumura, Y.; Pitout, J. D., Recent advances in the laboratory detection of carbapenemase-producing Enterobacteriaceae. Expert review of molecular diagnostics 2016, 1-12.

14. Tijet, N.; Boyd, D.; Patel, S. N.; Mulvey, M. R.; Melano, R. G., Evaluation of the Carba NP test for rapid detection of carbapenemase-producing Enterobacteriaceae and Pseudomonas aeruginosa. Antimicrobial agents and chemotherapy 2013, 57 (9), 4578-4580.

15. Bogaerts, P.; Yunus, S.; Massart, M.; Huang, T.-D.; Glupczynski, Y., Evaluation of the BYG Carba test, a new electrochemical assay for rapid laboratory detection of carbapenemase-producing Enterobacteriaceae. Journal of clinical microbiology 2016, 54 (2), 349-358.

16. Lupo, A.; Papp-Wallace, K. M.; Sendi, P.; Bonomo, R. A.; Endimiani, A., Non-phenotypic tests to detect and characterize antibiotic resistance mechanisms in Enterobacteriaceae. Diagnostic microbiology and infectious disease 2013, 77 (3), 179-194.

17. Nordmann, P.; Poirel, L.; Carrër, A.; Toleman, M. A.; Walsh, T. R., How to detect NDM-1 producers. Journal of clinical microbiology 2011, 49 (2), 718-721.

18. Miriagou, V.; Cornaglia, G.; Edelstein, M.; Galani, I.; Giske, C.; Gniadkowski, M.; Malamou-Lada, E.; MartinezMartinez, L.; Navarro, F.; Nordmann, P., Acquired carbapenemases in Gram-negative bacterial pathogens: detection and surveillance issues. Clinical microbiology and infection 2010, 16 (2), 112-122.

19. Poirel, L.; Walsh, T. R.; Cuvillier, V.; Nordmann, P., Multiplex PCR for detection of acquired carbapenemase genes. Diagnostic microbiology and infectious disease 2011, 70 (1), 119-123.
20. Schrader, C.; Schielke, A.; Ellerbroek, L.; Johne, R., PCR inhibitors-occurrence, properties and removal. Journal of applied microbiology 2012, 113 (5), 1014-1026.

21. Hedman, J.; Rådström, P., Overcoming inhibition in real-time diagnostic PCR. PCR detection of microbial pathogens 2013, 17-48.

22. Yang, S.; Rothman, R. E., PCR-based diagnostics for infectious diseases: uses, limitations, and future applications in acute-care settings. The Lancet infectious diseases 2004, 4 (6), 337-348.

23. Findlay, J.; Hopkins, K. L.; Meunier, D.; Woodford, $\mathrm{N}$., Evaluation of three commercial assays for rapid detection of genes encoding clinically relevant carbapenemases in cultured bacteria. Journal of Antimicrobial Chemotherapy 2015, 70 (5), 1338-1342.

24. Burckhardt, I.; Zimmermann, S., Using matrixassisted laser desorption ionization-time of flight mass spectrometry to detect carbapenem resistance within 1 to 2.5 hours. Journal of clinical microbiology 2011, 49 (9), 33213324.

25. Hrabák, J.; Chudáčková, E.; Papagiannitsis, C., Detection of carbapenemases in Enterobacteriaceae: a challenge for diagnostic microbiological laboratories. Clinical Microbiology and Infection 2014, 20 (9), 839-853.

26. Notake, S.; Matsuda, M.; Tamai, K.; Yanagisawa, H.; Hiramatsu, K.; Kikuchi, K., Detection of IMP metallo- $\beta$ lactamase in carbapenem-nonsusceptible Enterobacteriaceae and non-glucose-fermenting Gram-negative rods by immunochromatography assay. Journal of clinical microbiology 2013, 51 (6), 1762-1768.

27. Domínguez, C. M.; Kosaka, P. M.; Sotillo, A.; Mingorance, J. s.; Tamayo, J.; Calleja, M., Label-free DNAbased detection of Mycobacterium tuberculosis and rifampicin resistance through hydration induced stress in microcantilevers. Analytical chemistry 2015, 87 (3), 14941498.

28. Lahiri, H.; Mishra, S.; Mana, T.; Mukhopadhyay, R., Discriminating unalike single nucleobase mismatches using a molecularly resolved, label-free, interfacial LNA-based assay. Analyst 2016, 141 (13), 4035-4043.

29. Sharma, H.; Mutharasan, R., hly A gene-based sensitive detection of listeria monocytogenes using a novel cantilever sensor. Analytical chemistry 2013, 85 (6), 32223228 .

30. Domínguez, C. M.; Ramos, D.; Mendieta-Moreno, J. I.; Fierro, J. L.; Mendieta, J.; Tamayo, J.; Calleja, M., Effect of water-DNA interactions on elastic properties of DNA selfassembled monolayers. Scientific Reports 2017, 7 (1), 536.

31. Wentland, E. J.; Stewart, P. S.; Huang, C. T.; McFeters, G. A., Spatial variations in growth rate within Klebsiellapneumoniae colonies and biofilm. Biotechnology progress 1996, 12 (3), 316-321.

32. Huber, F.; Lang, H. P.; Glatz, K.; Rimoldi, D.; Meyer, E.; Gerber, C., Fast diagnostics of BRAF mutations in biopsies from malignant melanoma. Nano Letters 2016, 16 (9), 5373-5377.

33. Domínguez, C. M.; Kosaka, P. M.; Mokry, G.; Pini, V.; Malvar, O.; del Rey, M.; Ramos, D.; San Paulo, A. 1.; Tamayo, J.; Calleja, M., Hydration induced stress on DNA monolayers grafted on microcantilevers. Langmuir 2014, 30 (36), 10962-10969.

34. Mertens, J.; Rogero, C.; Calleja, M.; Ramos, D.; Martín-Gago, J. A.; Briones, C.; Tamayo, J., Label-free 
detection of DNA hybridization based on hydration-induced tension in nucleic acid films. Nature nanotechnology 2008, 3 (5), 301.

35. Wu, G.; Ji, H.; Hansen, K.; Thundat, T.; Datar, R.; Cote, R.; Hagan, M. F.; Chakraborty, A. K.; Majumdar, A., Origin of nanomechanical cantilever motion generated from biomolecular interactions. Proceedings of the National Academy of Sciences 2001, 98 (4), 1560-1564.

36. del Rey, M.; da Silva, R. A.; Meneses, D.; Petri, D. F.; Tamayo, J.; Calleja, M.; Kosaka, P. M., Monitoring swelling and deswelling of thin polymer films by microcantilever sensors. Sensors and Actuators B: Chemical 2014, 204, 602-610.

37. Girlich, D.; Poirel, L.; Nordmann, P., Value of the modified Hodge test for detection of emerging carbapenemases in Enterobacteriaceae. Journal of clinical microbiology 2012, 50 (2), 477-479.

38. Sushko, M. L.; Harding, J. H.; Shluger, A. L.; McKendry, R. A.; Watari, M., Physics of nanomechanical biosensing on cantilever arrays. Advanced Materials 2008, 20 (20), 3848-3853. 